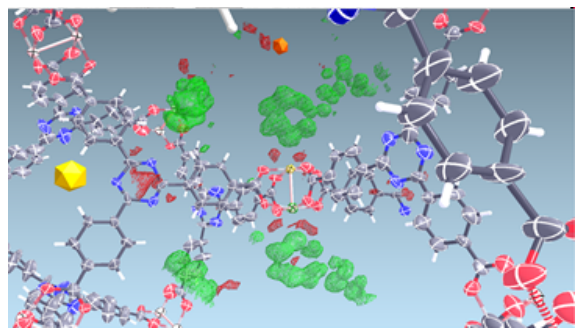

Figure 1. Experimental electron density of nicotine included inside the MOF known as PCN6 [3].

Keywords: crystal engineering, MOF, inclusion compounds

\section{MS33-O2 Solid-state transition-metal photoactive materials - tracing structure-property relationships via combined spectroscopic and crystallographic approaches}

Katarzyna N. Jarzembska ${ }^{1}$, Radosław Kamiński ${ }^{1}$

1. Biological and Chemical Research Centre, Department of Chemistry, University of Warsaw, Żwirki i Wigury 101, 02-089 Warsaw, Poland

email: katarzyna.jarzembska@gmail.com

Coordination complexes can be described as 'hot' small molecules due to their rich electronic and luminescent properties. $^{1}$ Especially interesting here are $\mathrm{d}^{8}$ or $\mathrm{d}^{10}$ transition-metal systems, which frequently constitute the active sites of both biological and chemical catalysts, have versatile applications in solar-energy conversion and other fields ranging from molecular electronics and photocatalysts to light-emitting diodes (LEDs) and biolabels. It is, thus, of high importance to sensibly control optical properties of such materials at the molecular level, in order to apply this knowledge to generate materials with particular properties across all the size scales from molecules to bulk materials, relevant for specific applications.

It occurs that short metal-metal contacts usually determine the nature of the lowest lying emissive states, and so are crucial to understand physical properties of the respective materials. In this contribution we shall present our most recent results regarding the analysis of charge transfer processes and structure-property relationships of selected coinage metal complexes $(\mathrm{Cu}, \mathrm{Ag}, \mathrm{Pt}, \mathrm{Ni}$, $\mathrm{Fe}$, etc.) in the solid state. ${ }^{2-5}$ For the purpose of our investigations we combined advanced spectroscopic and crystallographic techniques supported by theoretical calculations.

(1) Yam, V. W.-W.; Lo, K. K.-W. Chem. Soc. Rev. 1999, 28, 323. (2) Jarzembska, K. N.; Kamiński, R.; et al. Inorg. Chem. 2014, 53, 10594; Koshevoy, I. O.; Shakirova, et al. Dalton Trans. 2011, 40, 7927. (3) Filatov, A. S.; Hietsoi, O.; Sevryugina, Y.; Gerasimchuk, N. N.; Petrukhina, M. A. Inorg. Chem. 2010, 49, 1626. (4) Trzop, E.; Fournier, B.; Jarzembska, K.; Sokolow, J.; Kaminski, R.; et al. Acta Cryst. 2014, A70, C776. (5) Jarzembska, K. N.; Chen, Y.; et al. Phys. Chem. Chem. Phys. 2014, 16, 15792.

Keywords: coordination complexes, charge transfer, photocrystallography 